\title{
The Musicality of 140: Tempo, Rhythm and Structure
}

\author{
Jordan Browne
}

\begin{abstract}
This paper explores relationships between video games and music through a close reading of the minimalistic platform game 140 (Carlsen, 2014). Of particular interest to this investigation are concepts of tempo, rhythm and structure, and how these ideas can be extended to discuss the immediate case study as well as video games as a medium. Most importantly, this analysis is concerned primarily with these elements in a performative, spatio-temporal sense as opposed to an expression of sonic qualities.
\end{abstract}

Comparisons between video games and other forms of media, while certainly valuable, can become problematic as the interactive nature of games is inherently unique. While cinema or literary texts can be seen as interactive, it is the explicit nature of the interactivity that games manifest which sets them apart from other disciplines. It is in this sense, that commonality can be found between games and music through the act of play.

140 facilitates a unique dialogue on this topic as it is a game that is intensely musical while also functioning outside of some of the precincts of traditional music games, providing a distinctive lens for analysis without being distracted by its own aesthetics. Evidence of this can be seen in the game's design, aesthetics, mechanics and spatiality - a game where the player becomes part of a greater performance, enacting the musicality of space through play.

Keywords: Play, rhythm, tempo, creative gaming, space, explicit interactivity. 


\section{Introduction}

As a multidisciplinary field, approaches to theorising games are so often colonised from other areas of academia. Without delving too deeply into the ludology debates of a decade prior (e.g., Eskelinen, 2001; Juul, 2001), it seems fair to say that given the range of perspectives with vested interests in the medium, there are a number of viable methodologies that can be employed in the study of games - different contexts that demand different lenses of analysis. Colloquially, games are often compared to cinema which on the surface makes sense given the industry's best efforts to embody the familiar visual and narrative devices of the form. While there may be merits in thinking about games in this way, there is a fundamental rift in the mediums that makes any parallels drawn unavoidably problematic: the interactive nature of games. All media can be considered interactive in some way (Manovich, 2001, p. 56) but the type of interactivity that video games embody is explicit, requiring active participation unique from that of literature and cinema (Zimmerman, 2010). Films may be interactive but primarily in terms of Manovich's notion of reader effects; the cognitive process of interpreting a work (2001). Additionally, what Zimmerman outlines as functional interactivity also applies: the utilitarian interaction with a text that interfaces engagement without reconfiguration. The kind of interactivity that games embody requires the input of the player to actively participate in meaningful engagement, the "non-trivial effort" that Aarseth describes as "ergodic" (1997).

Amongst these observations, it is striking that games aren't more often considered through a lens of music (particularly that which is performed) since this medium can be analogous in its form of interactivity. This is not to say that comparison between games and other forms of media that are not explicitly interactive shouldn't exist, only that there is an inherent level of dissonance between these disparate mediums. Given these considerations and understanding the value of insights afforded by disciplines on the periphery of game studies, it is intriguing that the ways in which we play games may share more in common with music than many of the literary and film studies fields that are often drawn from. This paper seeks to explore some of these relationships between video games and music in reference to the minimalistic platform game 140 (Carlsen, 2014) - a work that bridges ideas fundamental to the understanding, experience and interpretation of music with ways we can theorise the spatiality and construction of video games.

It is important to distinguish that this discussion is not primarily concerned with the sonic properties of music - audio can be taken as one of many components in a wider spatial composition that includes all aspects of a game, from aesthetics to level design, to gameplay and mechanics. In this sense, it can be suggested that interpreting the musicality of a game is not so much about how a game sounds or the qualities of its audio but rather how the act of play within a virtual space can draw on ideas broadly found in music as a lens for analysis. Concepts such as rhythm, structure, meter, tempo and timing are all observable elements that can be used as analogies for play. It is also possible that some of these characteristics may extend beyond analogy to synonymy - timbre or melody may be difficult to compare directly to the way games are played whereas concepts such as rhythm correlate more distinctly between the two forms. These considerations can easily become blurred when the audiovisual aesthetics of a game mimic those of music or performance. 
There is, for example, very little comparison to be made between a game like Guitarooman (Koei, 2002) and actually playing a guitar: the physical interactions of pressing buttons on the controller as well as the ways in which players engage with the space of the game are fundamentally different to the experience of playing an actual guitar. This is not to say the game doesn't express its own rhythms, only that they may be dissonant with the perceived aesthetic.

\section{0 as a case study}

140 is a game developed by Jeppe Carlsen, a designer of Limbo (Playdead, 2010) fame with a talent for crafting unique and affective spaces. Besides the arbitrary fact that both games are platformers, there is little comparison to be made aesthetically or thematically between 140 and the developer's prior works: Carlsen trades the haunting, monochromatic landscapes of Limbo for psychedelic, electronic abstraction as different in pace as it is in visual styling. 140 plays like a conventional platformer, focusing on simple mechanics that primarily involve moving and jumping. What makes the game unique is that agents of the space are timed to a set tempo, affording a sense of rhythm and synchronicity that breathes life into the work. While the game itself isn't particularly long, a significant amount of content is packed into its three stages, with new ideas and mechanics evolving naturally as the game progresses. 140 is a visceral, rhythmic experience and in no uncertain terms, a video game that expresses a sense of musicality throughout all areas of its design.

140 is an excellent example for analysis because it is a work that escapes the traditional definitions of what constitutes a 'music game', while facilitating an experience that is undoubtedly musical. Pichlmair and Kayali (2007) outline several shared qualities of music games that they broadly categorise within the genres of rhythm games and instrument games. Rhythm games promote "little freedom of expression apart from the prerogative to perform" (Pichlmair \& Kayali, 2007), for example, timing button presses to enact a preconfigured score. In contrast, instrument games, while also confined by rulesets, offer the potential for play beyond a predetermined sequence where "the game provides - or at least pretends to provide - all the freedom of expression that a musical instrument calls for" (Pichlmair \& Kayali, 2007). This could include the player being empowered to compose an original melody or shape a soundscape that that exists outside of a scripted level or set of ordered inputs. While elements of these proposed classifications certainly apply to 140, the game is distinct among existing works, relieved of many of the trappings that traditionally come with this territory. Foremost, 140 is not about the aesthetic mimicry of playing an instrument that is embodied by games like Rockband (Harmonix, 2007) or Guitar Hero (Harmonix, 2005). Additionally, the agency of the player is fundamentally different to games such as Vib Ribbon (NanaOn-Sha, 2000) where the aesthetic of a platformer is given but interaction succumbs to a level of quantisation similar to more traditional rhythm games. 140 also stands apart from instrument games such as Electroplankton (Indieszero, 2005) in that gameplay is not concerned with the production of sound. Categorically, it seems 140 may have more in common in Super Mario Bros. (Nintendo R\&D4, 1985) than it does with the aforementioned examples. Despite the differences with other music games that can be identified, 140 is clearly musical with particular substance in its audiovisual aesthetic, tempo, rhythm and structure. 


\section{Tempo}

The aesthetics of the game's audio can be broadly categorised as electronic dance music (EDM), embodying tendencies of a number of subgenres such as techno and dubstep. This kind of music is most often defined by its rhythm, repetition and adherence to a quadruple meter with other typical traits including the use of syncopated rhythms, shuffled hi-hats and use of minor keys to establish tension (Bradley, 2013, p. 11). The game's name itself bares the first sign of its intentions beyond symbolically resembling the changing forms of the player, 140 beats per minute is the ubiquitous tempo of the dubstep genre. Although slight variation exists, this consistency is a trait necessitated by the performative aspects: live mixing and DJing is facilitated by a constant tempo as transitions between tracks are eased when songs share tempos that are inherently compatible. The name 140 then serves two purposes, the first being a nod to the aesthetic of its audio, the second being an indication of the tempo maintained sonically and through the spatiality of its world. It is this observation that makes the musicality of 140 distinctly unique from other music games: the world of the game is tempoed as opposed to the actions of the player themselves - the agents of this space meticulously timed to enact their behaviours on the appropriate measures - game objects dance in time with music, the space driven by this underlying premise.

Familiar tropes of traditional platform games make appearances within the context of this tempoed space. Platforms move in time with electronic samples, obstacles reconfigure themselves precisely on specific beats, and spring pads propel the player upwards in synchronisation to the repetitions of short drum loops. All aspects of the space in 140 act in time with this constant tempo, reinforcing one another through this adherence and exemplified by the aesthetics of the game's audio. What is interesting about this mechanical, mathematical precision of the space is that the player's actions are relieved of rigid instruction while simultaneously being subject to it. Where the environment is regulated, the player has the agency to move freely, abstracted from the synchronicity of their surroundings with an agency to act at any given moment irrespective of the temporal structure of the spatial composition.

\section{Quantisation}

Although the inputs of the player are not bound to the tempo of the game, play maintains rhythm in that in order to progress, the player is required to perform within the rhythms of the space. In games bound to an identifiable tempo, the player's actions are often quantised to the rest of the game. The result of player's input in Guitar Hero is perceivably immediate, but most often isn't as the outcome of input is delayed or moved forward to sync with the nearest beat. The consequence of this is that the player is not required to perform perfectly in time as their approximations are quantised to the correct measures in order to synchronise with the tempo of the music.

What is significant about 140 is that everything within its space is quantised while the player is given freedom to operate independently of this quantisation, moving through, around, with and against this space, mindful of but not restrained by its rules. It is interesting that while movement itself is uncontained, other consequences 
of the player's actions often are. There are a large number of checkpoints throughout the game, one before each individual obstacle or section. The player can be reset back to one of these checkpoints in a number of ways, mostly by colliding with a danger, normally signified by areas of mutating static. Although the player plummeting into a pit or area of danger in itself is free from quantisation, the reset sequence is timed with the tempo of the game so that there is a smooth transition in respawning the player without disrupting the space's tempo.

\section{Rhythm}

The consistent tempo of 140 is an observable element of the game that makes it unique, one that certainly inspires discussion around the musicality of its space. It is however, the effects of adhering to this tempo on play itself that are the most intriguing, and consequently revealing, aspects of this case study: the rhythms that this space generates. Rhythm in itself can be a difficult term to define with a plethora of definitions as well as applications on offer in varying contexts. Perhaps more so than some of the other terms appropriated from music theory and production utilised in this paper, discourse surrounding rhythm exists removed from the study of both music as well as video games. The use of Lefebvre's (1992) frames to describe linear and cyclical rhythms of video game space and structure, for example, could be employed to further elaborate the points raised in this section. In an attempt to maintain the scope of this paper however, a more traditional approach allows discussion to focus towards rhythm in the sense of musical structure as opposed to a broader philosophical perspective and the socio-political connotations that these approaches tend to entail. Most simply and suitably, an inherent priority of rhythm can be seen as "the perception of early events in a sequence generates expectancies concerning later events in real time" (Martin, 1972, p. 503). Less concerned with auditory associations of the concept, understanding rhythm as anticipation through what is perceived is an inclusive and useful way of thinking about the term in a way that is translatable to the context of games.

The rhythms of 140's space exist independent of the player who is unable to command of its spatiality or musicality; they are free from it but equally subject to it. The game is deeply aware of its mechanics, levels designed to its musicality across moment to moment gameplay. This directive makes itself apparent from the first level where a floating orb, in time with a single snare hit, changes its position on the third beat of each measure. The orb must be collected to progress and in order to do so, the player is required to time their movements with the game's tempo in anticipation of the approaching change. This notion is solidified in the subsequent section where platforms appear and disappear, again, on the snare hit of each measure. If the player jumps once the platform materialises, it will have disappeared before they land, resulting in the player falling to the static below. Traversing this segment (and consequently, the rest of the game) demands foresight to any visual changes; actions must be performed with the musicality of the space; its rhythms, motifs and repetitions. As the game progresses, the basic mechanics of well-timed jumps and the avoidance of obstacles are expanded but remain equally connected to the rhythms of the game and in order to navigate these spaces successfully, the player is required to understand the temporal and rhythmic structures that this space affords, 
acting in anticipation to events. The player's input becomes part of a wider composition, a single voice coordinated within a greater ensemble.

\section{Level structure}

A defining feature of level structure in 140 is the juxtaposition between calm, slow paced sections that evolve into faster, more hectic segments. The more subdued moments are low-key both sonically as well as in terms of gameplay, giving the player an opportunity to explore the space without any real challenge or obstacle. Upon activating the appropriate coloured orb, the player is able to transition levels into more intense states where music and gameplay both evolve. This pattern is repeated throughout most of the game and becomes the structural pillar of the game's composition.

A key characteristic EDM and dubstep in particular, is the build-up in intensity towards a song's climax. No matter how deep the bass line or punchy the snare hits, it is the promise of the breakdown or drop that has become a hallmark of the genre; the building of texture and rhythm signalling a shift in direction, ferocity and style. This is achieved not through an increase in tempo however, but through the use of half-time rhythms in preceding sections to give the impression of momentum and acceleration without manipulating the length of measures (Herrington, 2015, p. 10). A sense of space is established by these half-time passages where drums are sparse, emphasising texture and the role of electronic bass as both a melodic and rhythmic element. Double-time drums during drops can further emphasise a sense of escalation without altering tempo while affording the illusion of doing so. Structurally, 140 emulates these aesthetics of its sonic qualities through its design at a macro level - calmer, slow paced sections are methodical and absorbing while drops recontextualise space by escalating gameplay not only sonically, but also mechanically, introducing new elements in the form of more frequent and difficult challenges. This affords a double-time feeling that is expressed not just through the audio but through the act of play itself, reinforced by the fixed tempo of the space, allowing for smooth transitions between varying levels of intensity in design.

\section{Boss stages}

Beyond the repeated structure of individual levels, 140 also embraces another classical trait of platform games: boss stages - isolated sections where the player is required to overcome a powerful adversary or challenge in order to progress. These stages take may take different forms depending on the game but often build upon core mechanics in scripted or sequenced events. Additionally, a common directive for boss stages is to present the player with a challenge that builds on expectations of the prior stage or one that recontextualises familiar approaches in new ways. For example, boss encounters in Sonic the Hedgehog (Sonic Team, 1991) involve various conflicts with large robotic opponents piloted by the game's antagonist. The player advances by damaging these adversaries through jumping and spinning into their weak points. Proceeding this, the player is then required to avoid oncoming attacks before the opportunity to counter-attack once again arises. These boss stages are cohesive with the rest of the game as the primary mechanics of these 
encounters involve jumping and avoiding - elements which are foundational to the overall construction of the game.

Conversely, some games opt for an approach more abstracted from the moment to moment gameplay that the player may be familiar with. Alex Kidd in Miracle World (Sega, 1987) for example, is a platform game where the player jumps, punches and shoots their way through a variety of arcade style levels (not dissimilar to Sonic the Hedgehog). Based on the nature of the play, it would be fair to expect that boss stages would take an action orientated approach with an emphasis on jumping and avoidance. Instead the player enters into a sequence where they are unable to move, locked into participating in a rock-paper-scissors contest to progress. This mechanic, while fulfilling the change in pace that boss fights tend to embody, stands out as being incongruent to the greater experience of the game. The effectiveness of such methods can be judged on their own merits but what this example demonstrates is a tension in making a boss stage different and challenging while constructing an encounter that is also coherent to the wider tone of the game.

140 strikes an interesting balance along this spectrum of boss stages as gameplay dramatically shifts in direction during these moments while remaining contextualised by the rhythms of the space. The first encounter bares more resemblance to a vertical shooter than a platform game - the player enters a confined area, confronted by a floating mass of static above them. Upon entry to this stage the player is given a short window of space to experiment with the introduction of a new mechanic: a floating triangle that follows the player, firing a focused beam upwards on the first beat of every second bar. The goal of this encounter is fairly straightforward: the player needs to align themselves with the enemy above at the right time in order for one of the projected beams to hit. The stage becomes increasingly complicated as the boss multiplies on each successful hit, eventually escalating the frequency and difficulty of its own movement and firing patterns. What is interesting about this boss stage is that before this point there are not any encounters that involve damaging enemies and the mechanic of the floating triangle does not appear anywhere prior. On the surface, this may seem like an Alex Kidd moment - a change so different from the rest of the game that it risks becoming a detriment. However, while this approach deviates from expectation, these mechanics function effectively as they are contextualised by the temporal and rhythmic nature of the space. Timing movement to position the player correctly relies once again on playing to the tempo of the game, the aiming and positioning of the beams fired in direct conversation with rhythms of play communicated by audiovisual components.

A different implementation of these same mechanics could seem dissonant to the rest of the game if it were not for the intuition afforded by the game's tempo. Later boss stages also divert from expectation such as a flying section where the player avoids oncoming obstacles as well as a later recurrence of the shooting triangle in a more puzzle based approach that further abstracts from the platforming sections the game typically comprises of. It is in these moments that insight to the interactive nature of 140 is uncovered - it is not a work defined by a specific mechanic or genre stereotype, but rather, how all of the mechanics it utilises are unified by the tempo of the space. Any number of platforming or puzzle designs could be implemented 
naturally into the levels of the game, provided that they embrace the rhythms of its world.

\section{Extending this forward}

140 is a game that facilitates a rich discussion around concepts of rhythm, tempo, structure and play. The true value of this analysis however is not in the form of revelations specifically about the case study chosen but the insights afforded by this approach to thinking about video games. At the back of this dialogue, there is a question about 140 that begs consideration: is its musicality at all diminished if the game is played with the sound muted? The way rhythms are conveyed to the player would certainly be altered without the affordances of the game's soundscape - the presence of a continuous tempo and the mappings of mechanics to beats would undoubtedly become less obvious. On the other hand, the temporal progression of the game would remain unchanged. The agents of the space are indifferent to whether or not their mechanics are expressed sonically. If rhythm can be defined broadly as ordered characteristics of succession (Fraisse, 1982), then the rhythms of 140 can certainly be observed through visual stimuli and the experiential nature of play. Thus, in this instance at least, the perception of rhythm and tempo can be relieved of sonic qualities as well as analogy.

One of the reasons we are led to believe 140 is a music game is because of the explicit link between the game's sonic qualities and it's mechanics, when in fact, the game's tempo and rhythm supersede this connection (although they are most certainly supported by it). This realisation begs the consideration that if the musicality of 140 is afforded by play itself, then can this type of discussion be extended to games beyond typical aesthetics, as well as types of play, that would normally inspire this kind of investigation? What are the tempos and rhythms of Sonic the Hedgehog and how would they compare to Super Mario Bros. or Alex Kidd in Miracle World?

The programmatic nature of games ensures a sense of predictability and structure in that there is an inescapable tempo defined at the very least by the frame rate of a work and the basic loop of the game. Admittedly, judging a game by a quality that is so unobservable would be irrelevant to the analysis of any perceived rhythm but nonetheless, a precedent is set for understanding that games fundamentally are tempoed at the core of their design. Beyond technical aspects there is also a sense of rhythm afforded by the act of play itself. There is something undoubtedly rhythmic about fighting games such as the Tekken (Namco, 1994-2015) and Soulcalibur (Project Soul, 1996-2014) series. A more interesting challenge may be mapping these qualities to a game even further removed from the case study of this paper strategy games like Sid Meier's Civilization V (Firaxis Games, 2010) surely have some sort of tempo embodied by second to second interactions as well as the wider structures of turn based gameplay. As an extension of using these ideas as a framework for analysis, this approach may prove equally useful as a way of designing games. 140 is an example of a highly cohesive work in which all of the game's elements are tied to the tempo of the space. Developing games with their rhythms in mind could be an effective way to build systems and mechanics that are intelligible and complementary to a greater thesis - a ludic structure focused on play and pacing that supersedes purely genre or narrative intentions. 


\section{References}

Aarseth, E. J. (1997). Cybertext: Perspectives on Ergodic Literature. Baltimore, Md: Johns Hopkins University Press.

Eskelinen, M. (2001). The Gaming Situation. The International Journal of Computer Game Research, 1(1).

Bradley, L. (2013). Decoding Dubstep: A Rhetorical Investigation of Dubstep's Development from the Late 1990s to the Early 2010s. Florida State University. Retrieved from: http://diginole.lib.fsu.edu/uhm/163

Fraisse, P. (1982). Rhythm and Tempo. In Deutsch, D. (ed.), The Psychology of Music, 149-180. New York: Academic Press.

Herrington, J. (2015). Towards an interactive environment for the performance of Dubstep music. Edith Cowan University.

Juul, J. (2001). Games Telling stories? - a brief note on games and narratives. The International Journal of Computer Game Research, 1(1).

Lefebvre, H. (2008). Rhythmanalysis: Space, Time and Everyday Life. London: Continuum. (Original work published 1992)

Manovich, L. (2001). The Language of New Media. Cambridge, Mass: MIT Press.

Martin, J. G. (1972). Rhythmic (hierarchical) versus serial structure in speech and other behavior. Psychological Review, 79(6), 487-509.

Pichlmair, M. \& Kayali, F. (2007). Levels of Sound: On the Principles of Interactivity in Music Video Games. Situated Play, Proceedings of DiGRA 2007 Conference. Retrieved from: http://www.digra.org/dl/db/07311.14286.pdf

Zimmerman, E. (2010). Narrative, Interactivity, Play, and Games: Four naughty concepts in need of discipline. Retrieved from:

http://www.ericzimmerman.com/texts/Four_Concepts.html

\section{Games}

Carlsen, J. (2014). 140 [Computer game].

Firaxis Games. (2010). Sid Meier's Civilization V. 2K Games.

Koei. (2002). Guitarooman [Playstation 2 game]. THQ.

Harmonix. (2005). Guitar Hero [PlayStation 2 game]. RedOctane.

Harmonix. (2007). Rock Band [PlayStation 3 game]. Electronic Arts.

Indieszero. (2005). Electroplankton [Nintendo DS game]. Nintendo. 
Project Soul. (1996 - 2014). Soulcalibur [Video game series]. Bandai Namco Entertainment.

Namco. (1994 - 2015). Tekken [Video game series]. Bandai Namco Entertainment.

NanaOn-Sha. (2000). Vib Ribbon [Playstation game]. Sony Computer Entertainment.

Nintendo R\&D4. (1985). Super Mario Bros. [Nintendo Entertainment System Game]. Nintendo.

Playdead. (2010). Limbo [Xbox 360 game]. Microsoft Studios.

Sega. (1987). Alex Kidd in Miracle World [Sega Master System game]. Sega.

Sonic Team. (1991). Sonic the Hedgehog [Sega Mega Drive game]. Sega. 


\section{About the Author}

\section{Jordan Browne}

Jordan is a senior lecturer at Media Design School in New Zealand, teaching game studies, research and production. With an educational background in film and screen and media studies, Jordan is also a musician and indie developer with an interest alternative and experimental games that challenge the boundaries of the medium.

Contact:_jordnbrowne@gmail.com 


\section{The Journal of Creative Technologies (JCT)}

https://ctechjournal.aut.ac.nz

\section{ISSN: 2230-2115}

Colab, Auckland University of Technology, New Zealand

Creative Commons Attribution 4.0 International License (CC-BY)

All articles published in The Journal of Creative Technologies (JCT) from Issue 4 onwards are licensed under a Creative Commons Attribution 4.0 International License (CC-BY). The copyright of the material remains with the author(s), and third-parties are granted permission to use, shared, and adapted the material, provided the original work is appropriately attributed.

The Journal of Creative Technologies (JCT) is an online, open access, peer-reviewed journal for the publication of research and innovation about new technologies, creative practices, and critical theories. The journal aims to explore applied, methodological and theoretical perspectives on emergent technological platforms and strategies through thematically focused issues.

$J C T$ is a research communication platform published by Colab at the Faculty of Design and Creative Technologies, Auckland University of Technology. 